\title{
Does the Cyclosporine Still Have a Potential Role in the Treatment of Acute Severe Steroid-Refractory Ulcerative Colitis?
}

\author{
Chang Soo Eun and Dong Soo Han \\ Department of Internal Medicine, Hanyang University Guri Hospital, Hanyang University College of Medicine, Guri, Korea
}

See "Infliximab versus Cyclosporine Treatment for Severe Corticosteroid-Refractory Ulcerative Colitis: A Korean, Retrospective, Single Center Study" by Eun Hye Kim, et al. on page 601, Vol. 9. No. 5, 2015

Ulcerative colitis (UC) is a chronic inflammatory disease of the colon characterized by diffuse mucosal inflammation. Acute severe colitis, a potentially lethal condition requiring hospitalization and intensive medical treatment can develop in 18\% to $25 \%$ of patients with UC. Although intravenous corticosteroids are the mainstay of conventional medical treatment in this condition, about $30 \%$ to $40 \%$ of patients are resistant to steroids treatment. ${ }^{1}$ In the past time when other medical treatments were unavailable for steroid-refractory UC, the only option was an emergency colectomy, which had resulted in about 10\% of 3-month mortality rate after surgery. Over the recent past, both cyclosporine and infliximab have been shown to have some benefits for severe UC patients who are refractory to intravenous corticosteroids.

Since 1994, through several controlled and noncontrolled clinical trials, intravenous cyclosporine, a calcineurin inhibitor, has been shown to be efficacious in severe attacks of UC patients with $64 \%$ to $82 \%$ of response rates. The rapid onset of action is a major advantage of cyclosporine therapy, which enables timely colectomy to be performed in patients with nonresponse. However, cyclosporine therapy has been associated with high risk of long-term colectomy (88\% of colectomy rate at 7 years). UC patients already exposed to thiopurines prior to acute severe attacks are at increased risk of colectomy. Furthermore, significant risk of toxicity and side effects such as nephrotoxicity, hypertension, seizures and neurotoxicity as well as rigorous drug monitoring limit its use in clinical practice.

In contrast, since 2005, several randomized placebo-controlled clinical trials demonstrated that infliximab, a monoclonal antibody against tumor necrosis factor $\alpha$, was safe and ef- fective in severe steroid-refractory UC patients with $60 \%$ to $80 \%$ of short-term avoiding rates of colectomy. Infliximab also has a long half-life and intensive drug monitoring is not required. Hence, infliximab has been widely used as a pivotal option in patients with acute severe steroid-refractory UC. ${ }^{2}$

So, should we discard cyclosporine in favor of infliximab in these patients? Although there have been several data comparing the efficacy of intravenous cyclosporine and infliximab, it is still debated which medication is more effective in steroidrefractory UC., ${ }^{3,4}$ At present, some guidelines recommend intravenous cyclosporine or infliximab as a medical rescue therapy in acute severe UC patients who is refractory to intravenous steroids. Recent meta-analysis of six retrospective cohort studies compared the clinical outcomes of steroid-refractory UC patients receiving infliximab or cyclosporine as a rescue therapy showed no significant differences in the 3-month and 12-month colectomy rate, in adverse events, and in postoperative complications between two groups. ${ }^{5}$ In addition, the first prospective randomized controlled trial comparing the efficacy of cyclosporine versus infliximab, recently published in Europe, demonstrated there is no significant difference in the treatment outcomes between two groups. ${ }^{6}$

However, most of these studies have been performed in Western countries. Compared with Western population, Korean UC patients have distinct genetic and ethnic backgrounds as well as different environmental characteristics such as diet and intestinal microbial change. ${ }^{7,8}$ To date, there has been no data comparing the efficacy of cyclosporine versus infliximab in steroidrefractory UC patients from Korea.

In this issue of Gut and Liver, Kim et al. ${ }^{9}$ investigated the ef-

Correspondence to: Dong Soo Han

Department of Internal Medicine, Hanyang University Guri Hospital, Hanyang University College of Medicine, 153 Gyeongchun-ro, Guri 471-701, Korea

Tel: +82-31-560-2226, Fax: +82-31-555-2998, E-mail: hands@hanyang.ac.kr pISSN 1976-2283 eISSN 2005-1212 http://dx.doi.org/10.5009/gnl15293

(a) This is an Open Access article distributed under the terms of the Creative Commons Attribution Non-Commercial License (http://creativecommons.org/licenses/by-nc/4.0) which permits unrestricted non-commercial use, distribution, and reproduction in any medium, provided the original work is properly cited. 
ficacy of cyclosporine and infliximab in 43 patients with severe steroid-refractory UC in a retrospective manner. They compared clinical outcomes of 10 patients who had received intravenous cyclosporine with those of 33 patients who had received infliximab for acute severe steroid-refractory UC. The authors showed that there was no difference in preventing colectomy during follow-up of 12 months (primary outcome) between cyclosporinetreated group and infliximab-treated group. Meanwhile, in the subgroup analysis, infliximab with azathioprine was superior to cyclosporine for preventing colectomy (hazard ratio of infliximab with azathioprine compared to cyclosporine only, 0.073; 95\% confidence interval, 0.008 to 0.629). In terms of secondary outcomes, length of hospital stay after rescue therapy was shorter in the infliximab-treated group, and the rates of adverse events did not differ between two groups. This study, firstly compared the efficacy of rescue therapy in Korean patients with acute severe steroid-refractory UC, provide an essential insight into the treatment options available for these patients in Korea.

However, as the authors described, this study performed retrospectively with a small sample size and compared the clinical outcomes between groups enrolled from different periods of time. And, the better outcome for avoiding colectomy in the infliximab group relative to the cyclosporine group might be resulted from a beneficial interaction of infliximab and azathioprine. To validate the results of this study, a prospective multicenter randomized clinical trial comparing the efficacy of cyclosporine versus infliximab in Korean steroid-refractory UC patients is warranted.

Acute severe UC patients who are resistant to intravenous steroids continue to be a potentially life threatening condition in clinical practice. At present, it is not clear whether infliximab therapy is more effective in acute severe steroid-refractory UC patients than intravenous cyclosporine therapy. Therefore, in the absence of definite contra-indication to a particular therapy, the individual situation of each patient and clinician's experience should be considered when deciding treatment option for rescue therapy. In addition, clinicians have to consider that even if rescue therapy with either infliximab or intravenous cyclosporine is clinically important in acute severe steroid-refractory
UC, medical treatment should not postpone the decision for colectomy in patients with inadequate response to rescue therapy.

\section{CONFLICTS OF INTEREST}

No potential conflict of interest relevant to this article was reported.

\section{REFERENCES}

1. Park SC, Jeen YT. Current and emerging biologics for ulcerative colitis. Gut Liver 2015;9:18-27.

2. Seo HI, Park DI, Kim TO, et al. The effect of infliximab on patients with ulcerative colitis in Korea. Intest Res 2014;12:214-220.

3. Sjöberg M, Walch A, Meshkat M, et al. Infliximab or cyclosporine as rescue therapy in hospitalized patients with steroid-refractory ulcerative colitis: a retrospective observational study. Inflamm Bowel Dis 2012;18:212-218.

4. Croft A, Walsh A, Doecke J, Cooley R, Howlett M, Radford-Smith G. Outcomes of salvage therapy for steroid-refractory acute severe ulcerative colitis: ciclosporin vs. infliximab. Aliment Pharmacol Ther 2013;38:294-302.

5. Chang KH, Burke JP, Coffey JC. Infliximab versus cyclosporine as rescue therapy in acute severe steroid-refractory ulcerative colitis: a systematic review and meta-analysis. Int J Colorectal Dis 2013; 28:287-293.

6. Laharie D, Bourreille A, Branche J, et al. Ciclosporin versus infliximab in patients with severe ulcerative colitis refractory to intravenous steroids: a parallel, open-label randomised controlled trial. Lancet 2012;380:1909-1915.

7. Han DS. Current status and prospects of intestinal microbiome studies. Intest Res 2014;12:178-183.

8. Jung YS, Song CS, Kim ER, et al. Seasonal variation in months of birth and symptom flares in Korean patients with inflammatory bowel disease. Gut Liver 2013;7:661-667.

9. Kim EH, Kim DH, Park SJ, et al. Infliximab versus cyclosporine treatment for severe corticosteroid-refractory ulcerative colitis: a Korean, retrospective, single center study. Gut Liver 2015;9:601606. 